\title{
Noticias del nutritionDay
}

\author{
nutritionDay News \\ Angélica María Pérez, ND, Esp. MSc (c) \\ Coordinadora Nacional nutritionDay, Asociación Colombiana de Nutrición Clinica
}

https://doi.org/10.35454/rncm.v3n2.204

Resumen de la participación de Colombia en el nutritionDay 2019.

- Colombia participó en 2019 por décimo año consecutivo en el nutritionDay con 1.881 pacientes de medicina interna, cirugía, oncología, críticos y geriátricos de los cuales $46 \%$ fueron mujeres. En la Tabla 1 se muestra la participación de los países latinoamericanos.

- Se observaron resultados importantes como que la mayoría de hospitales y servicios cuentan con una estrategia de cuidado nutricional ( $84 \%$ y $80 \%$ respectivamente). Sin embargo, solo $23 \%$ de los hospitales registra indicadores de calidad en el ámbito nacional o regional.

Tabla 1. Participación de países latinoamericanos en el nutritionDay

\begin{tabular}{|l|c|c|c|}
\hline \multicolumn{1}{|c|}{ País } & Hospitales & Servicios & Pacientes \\
\hline Argentina & 2 & 12 & 306 \\
\hline Brasil & 7 & 13 & 343 \\
\hline Colombia & 27 & 52 & 1.881 \\
\hline Ecuador & 3 & 8 & 108 \\
\hline Paraguay & 8 & 17 & 473 \\
\hline Total & & & 3.111 \\
\hline
\end{tabular}

En esta ocasión participaron más de 100 encuestadores colombianos que, bajo la dirección de los coordinadores de cada hospital le apuestan al mejoramiento continuo de sus servicios clínicos y de los grupos de terapia nutricional a lo largo del territorio colombiano. De esta forma cada año se dedica un día a la nutrición con el firme propósito de crear cultura organizacional entorno a la nutrición clínica. Una vez más, muchas gracias a todas las instituciones que hacen parte de este estudio.

El reporte completo de los resultados de Colombia se puede descargar libremente en: https://www. nutritionday.org/cms/upload/pdf/6_about nutritionDay/6.9.1.national_report_2019/ CO_country_Report_onco_2019_en.pdf.

En esta sección presentamos resultados relevantes del nutritionDay 2019 en Colombia teniendo en cuenta las etapas del proceso de cuidado nutricional institucional según la Declaración de Cartagena, es decir, Detectar, Nutrir y Vigilar.

\section{DETECTAR}

El 61,6\% de los servicios hospitalarios realiza tamizaje nutricional a los pacientes al ingreso. Las herramientas más usadas son: MST, NRS 2002 y MUST. Se identificó que $17,6 \%$ de los pacientes presentan riesgo de malnutrición y 13,6 \% malnutrición al ingreso.

El 49,8\% de los pacientes había perdido peso en los últimos 3 meses y 33,4\% tenían reducida su ingesta la última semana antes del ingreso hospitalario.

\section{NUTRIR}

Durante la hospitalización 55,4\% de los pacientes recibían alimentación regular, 31,3\% de los pacientes estaban con dieta terapéutica, $7,9 \%$ con dieta fortificada, $13,5 \%$ recibían suplementación oral, 3,9\% nutrición enteral y $2,7 \%$ nutrición parenteral. Los requerimientos nutricionales son calculados y determinados por el nutricionista dietista de la unidad; sin embargo, solamente entre $42 \%$ y $44 \%$ de los pacientes tenían un plan de intervención definido para calorías o proteínas en la historia clínica. 
Durante la observación directa del almuerzo, $45 \%$ de los pacientes no consumió totalmente su dieta, de los cuales $8,1 \%$ no comió nada, lo cual ocurrió por las siguientes razones: el sabor de la comida, el olor o consistencia de los alimentos no fue de su agrado ( $16 \%$ y $10,3 \%$ respectivamente), falta de apetito $30,8 \%$, náuseas o vómito $12,1 \% .9,2 \%$ de los pacientes estaba sin vía oral por indicación médica (exámenes o cirugías pendientes).

\section{VIGILAR}

En promedio, los pacientes incluidos en el estudio tuvieron una estancia hospitalaria de 14 días.

En cuanto a los accesos nutricionales, las sondas para alimentación enteral más utilizadas son la sonda nasogástrica y la gastrostomía endoscópica percutánea.
A los 30 días del nutritionDay, se realiza control de los pacientes para determinar las condiciones de egreso hospitalario. Se encontró que $12,5 \%$ de los pacientes permaneció en la institución, $3 \%$ fue transferido a otra institución, $0,58 \%$ trasladado a unidades de cuidado crónico, $75,9 \%$ se encontraba en casa, mientras que $10 \%$ de los pacientes fue readmitido en la institución.

La participación en el nutritionDay 2020 permitirá obtener indicadores económicos y de calidad, retroalimentación y una evaluación comparativa de los datos. Con esta información, se podrá hacer seguimiento al proceso de cuidado nutricional hospitalario, y a mediano plazo reducir la brecha entre el conocimiento y la acción. Los esperamos, más información en nutritionday@ nutriclinicacolombia.org o celular: 3188895653.

\title{
Prepárate para participar en el nutritionDay
}

\author{
TALLER PRÁCTICO VIRTUAL \\ Angélica Pérez, coordinadora del nDay Colombia \\ Octubre 23, 2020. 4pm (GMT-5)
}

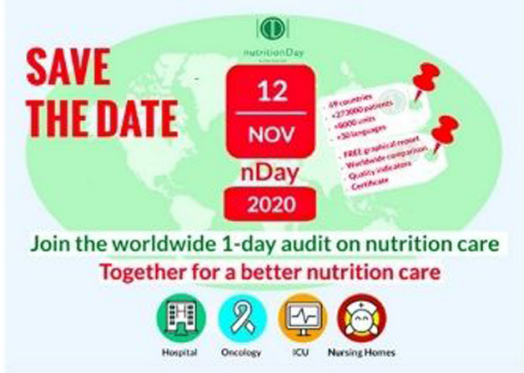

INFORMACIÓN E INSCRIPCIONES: nutritionday@nutriclinicacolombia.org 


\section{ÚLTIMAS PUBLICACIONES SOBRE EL NUTRITIONDAY}

\section{El riesgo nutricional se asocia al aumento de la mortalidad intrahospitalaria y a la reducción de la probabilidad de egreso al domicilio: resultados del estudio nutritionDay 2009-2015}

\section{Nutritional risk is associated with an increase of in-hospital mortality and a reduction of being discharged home: Results of the 2009-2015 nutritionDay survey}

Colombia publicó en la revista Clinical Nutrition ESPEN de junio de 2020 un estudio donde se evaluó el impacto del riesgo nutricional en los desenlaces clínicos ${ }^{(1)}$. Se mostró, después de un análisis multivariado, aumento en la mortalidad hospitalaria y una reducción del alta hospitalaria en los pacientes con riesgo de desnutrición (según la herramienta MST > = 2). Además, se observó que el riesgo nutricional sigue siendo de alta prevalencia en el mundo, lo que implica la necesidad de promover un cuidado nutricional óptimo y oportuno.

Para realizar este análisis se utilizó la base de datos del nutritionDay con 7.994 pacientes colombianos de 248 servicios hospitalarios y más de 155.000 pacientes en el mundo. Se demostró que la prevalencia de riesgo de desnutrición en Colombia fue 38 \%, 41 \% en Latinoamérica, y $32 \%$ en el mundo. La mitad de los servicios hospitalarios colombianos realizan tamizaje nutricional al ingreso, comparado con $80 \%$ en Latinoamérica y $62 \%$ en el mundo. Únicamente $23 \%$ de los pacientes colombianos identificados con riesgo nutricional recibían algún tipo de terapia nutricional. La Figura 1 muestra las principales herramientas utilizadas. El riesgo (hazard ratio) de mortalidad hospitalaria para los pacientes colombianos con riesgo de desnutrición fue 1,94 (95\% CI 1,53, 2,46; $\mathrm{p}<0,001)$ y el riesgo de egreso hospitalario a domicilio de 0,82 (95\% CI 0,76, 0,88; $\mathrm{p}<0,001$ ).

Estos resultados permiten mostrar el impacto del riesgo nutricional en los desenlaces de los pacientes y señalar la importancia de realizar un adecuado cuidado nutricional que conlleve a identificar a los pacientes en riesgo de desnutrición para administrar una terapia nutricional óptima y oportuna. La participación de países latinoamericanos en el nutritionDay es una oportunidad para aumentar el conocimiento y promover la importancia de la desnutrición hospitalaria ${ }^{(1)}$.

\section{Reducción de la brecha entre conocimiento y acción en la atención nutricional hospitalaria: desarrollo e implementación del nutritionDay 2,0}

\section{Reducing the knowledge to action gap in hospital nutrition care - Developing and implementing nutritionDay 2.0}

El equipo coordinador del nutritionDay en Viena, Austria, dirigido por el Profesor Hiesmayr, publicó un artículo en la revista Clinical Nutrition sobre el origen

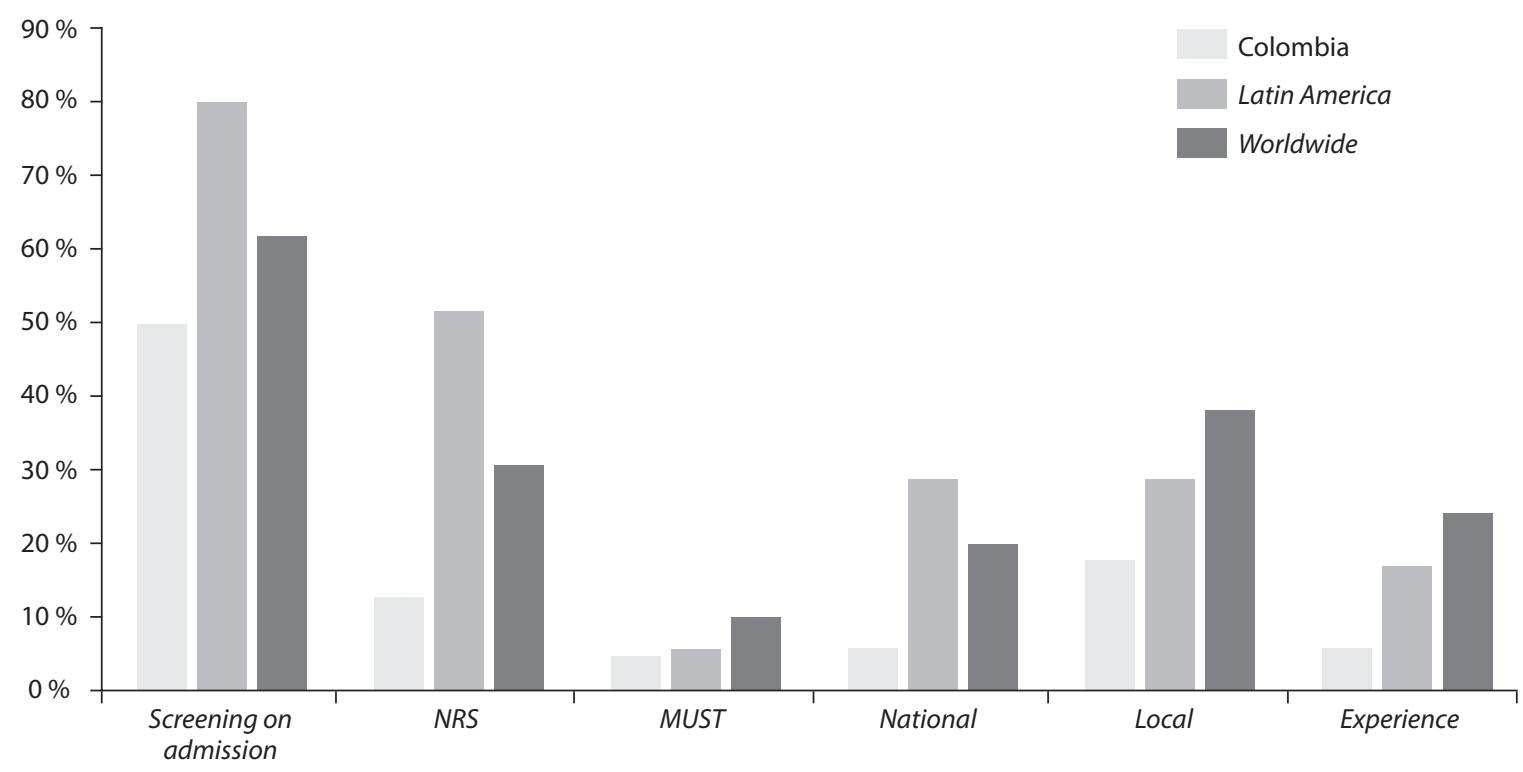

Figura 1. Prevalencia de tamizaje nutricional al ingreso hospitalario y herramientas utilizadas ${ }^{(1)}$. 
de los cuestionarios que se aplican desde el año 2016 $(n \text { Day } 2,0)^{(2)}$. El objetivo del cambio en los cuestionarios fue la necesidad de darle un nuevo enfoque al nutritionDay y así reducir la brecha del "conocimiento a la acción” en el cuidado nutricional. El artículo describe la estrategia, los métodos, los instrumentos y la experiencia que condujeron a desarrollar e implementar el $n$ Day 2.0. La versión $n$ Day 2.0 es una intervención de tipo auditoría que utiliza indicadores económicos y de calidad, retroalimentación, evaluación comparativa y estrategias de acción autodefinidas para reducir esta brecha en la atención nutricional hospitalaria. El proceso de desarrollo e implementación del nDay 2.0 durante tres años resultó en un nuevo cuestionario de auditoría basado en 36 indicadores económicos y de calidad de la atención nutricional a nivel de hospital, unidad y paciente, un nuevo informe de evaluación comparativa y retroalimentación orientada a la acción.

La evaluación del nutritionDay 2.0 está aún en curso e incluirá la satisfacción y la utilidad de las herramientas y los efectos a corto, mediano y largo plazo en esta brecha. Se espera que en la práctica clínica el nutritionDay 2.0 tenga el potencial de promover cambios de comportamiento para mejorar los resultados de la atención nutricional hospitalaria. En la investigación, los datos

\section{Referencias bibliográficas}

1. Cardenas D, Bermúdez C, Pérez A, et al. Nutritional risk is associated with an increase of in-hospital mortality and a reduction of being discharged home: Results of the 20092015 nutritionDay survey. Clin Nutr ESPEN. 2020;38:13845. doi:10.1016/j.clnesp.2020.05.014.

2. Moick S, Hiesmayr M, Mouhieddine M, et al. Reducing the knowledge to action gap in hospital nutrition care - Developing generados permitirán avanzar en el conocimiento sobre la desnutrición hospitalaria y la calidad de la atención nutricional $^{(2)}$.

\section{Exploración de los factores que influyen en la ingesta alimentaria durante la hospitalización: resultados del análisis de la base de datos de NutritionDay (2006-2013)}

\section{Exploring factors influencing dietary intake during hospitalization: Results from analyzing nutritionDay's database (2006-2013)}

El equipo de Kontogianni y colaboradores analizó la base de datos del nutritionDay, en el período 2006 - 2013, para explorar las razones de la reducción de la ingesta de alimentos y los factores asociados durante la hospitalización según lo informado por los pacientes ${ }^{(3)}$. Se mostró que únicamente 41,6 \% de los pacientes informó haber consumido toda la comida que les sirvieron, mientras que $9,3 \%$ no comió nada, aunque podían hacerlo. El estudio muestra que la ingesta de alimentos durante la hospitalización está asociada a variables relacionadas tanto con el estado del paciente (por ejemplo, clínico, físico) como con la calidad de la comida del hospital ${ }^{(3)}$.

and implementing nutritionDay 2.0. Clin Nutr. 2020;S02615614(20)30332-0. doi:10.1016/j.clnu.2020.06.021.

3. Kontogianni MD, Poulia KA, Bersimis F, et al. Exploring factors influencing dietary intake during hospitalization: Results from analyzing nutritionDay's database (20062013). Clin Nutr ESPEN. 2020;38:263-70. doi:10.1016/j. clnesp.2020.04.001. 